\title{
Die Entwicklung und Verbreitung der Urania zur Popularisierung der Astronomie
}

\author{
Gudrun Wolfschmidt \\ Schwerpunkt Geschichte der Naturwissenschaften,Mathematik und Technik, \\ Universität Hamburg
}

\begin{abstract}
The development of public outreach activities in Berlin rested on the archives of Alexander von Humboldt who gave 16 lectures in 1827/28 describing his travels for an auditorium of 13000 . In 1863 W. Foerster and E.Schoenfeld revived this idea to transfer knowledge in down-to-earth-lectures to the public by founding the society of the URANIA which was a name and later-on the denomination for a building too. This "scientific theater" also comprised a small observatory and the idea spread over all Germany and Vienna. In the economically difficult times around 1928 the Berlin Urania had to be shut down, the building was completely destroyed, some of instruments had been transferred to Berlin/Treptow but the rest was stolen.
\end{abstract}

\section{Einleitung}

Seit der Antike wurden in der Kunst und in den Wissenschaften abstrakte philosophische Begriffe, "ewige Wahrheiten" und menschliche Eigenschaften als lebende und handelnde Wesen dargestellt - personifiziert in weiblichen Allegorien. In der griechischen Mythologie ist die URANIA eine der neun Musen, die Muse der Sternkunde ${ }^{1}$ Einen entscheidenden Schritt zur Popularisierung machte bereits der Forschungsreisende Alexander von Humboldt (1769-1859)

\footnotetext{
${ }^{1}$ Die Musen: Die Muse Urania, Muse der Sternkunde, ihre Zeichen sind Himmelsglobus und Zeigestab.; Die Muse Kalliope, Muse der epischen Dichtung; Die Muse Thalia, Muse der komischen Dichtung; Die Muse Melpomene, Muse der tragischen Dichtung; Die Muse Terpsichore, Muse der Chorlyrik und des Tanzes; Die Muse Euterpe, Muse der Lyrik; Die Muse Polyhymenie, Muse der Hymnendichtung; Die Muse Erato, Muse der Liebesdichtung; Die Muse Clio, Muse der Geschichtsschreibung;.
} 


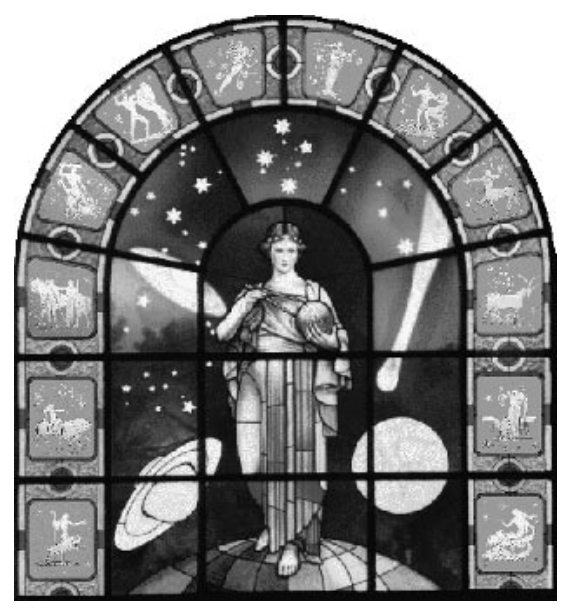

Abb. 1: Muse Urania http://www.muse-net.com/urania.gif

mit seinen Reisebeschreibungen und ab 1845 mit dem fünfbändigen monumentalen Werk "Kosmos". 1827/28 hielt er im Gebäude der Berliner Singakademie 16 öffentliche Vorträge, die sogenannten "Kosmos-Vorlesungen", die von rund 13.000 Zuhörern besucht wurden und ein großartiges gesellschaftliches Ereignis im königlichen Berlin bildeten. Ab 1844 folgten Vorträge im neugegründeten "Wissenschaftlichen Verein". Humboldts Vorträge über "physikalische Geographie" umfaßten Themen wie Astronomie, Biologie, Geowissenschaften, Naturphilosophie, Physik (Elektrizität, Magnetismus und Optik) sowie Chemie. Damit gab Humboldt entscheidende Impulse zur Wissenschaftspopularisierung. Hierdurch wurden wissenschaftliche Entdeckungen und technische Erfndungen der Öffentlichkeit zugänglich gemacht. Pro Vortrag waren mehr als 800 Zuhörer anwesend. Das soziale Spektrum der Hörer war weit gestreut, vom einfachen Handwerker bis zu König Friedrich Wilhelm III. (König von 1797 bis 1840).

Durch die steigende Anzahl populärer Pulikationen wurden relativ viele Personen zu eigenen Beobachtungen oder zur Herstellung astronomischer Instrumente angeregt, ein Beispiel wäre Wilhelm Herschel (1738-1822). Der Zuckerfabrikant und Bankier Wilhelm Beer (1797-1850) errichtete im Dachgeschoß seiner Villa im Tiergarten eine große Privatsternwarte mit dem Ziel, eine erste Karte der Oberflächen von Mars und Mond mit Hilfe neuer Beobachtungsmethoden zu erstellen. Solche Privatsternwarten standen einem eingeschränkten Besucherkreis zur Verfügung. 


\section{Die Urania in Berlin - Gründung und Aktivitäten}

Unter Bezugnahme auf die Vorleistungen Alexander von Humboldts bei seinen öffentlichen Vorträgen, die so begeistert aufgenommen wurden, plante man durch den Bau einer Sternwarte dem Wunsch nach Information über die neuesten Entwicklungen der Astronomie entgegenzukommen. Durch aufsehenerregende Entdeckungen z.B. neuer Planeten und Planetoiden war die Astronomie Anfang des 19. Jahrhunderts ins besondere Interesse einer breiten Öffentlichkeit geraten. Humboldt ließ daher der 1835 gegründeten Berliner Sternwarte unter Leitung von Johann Franz Encke (1791-1865)

"die bis dahin völlig neue Verpflichtung auferlegen ..., daß sie allmonatlich an etwa zwei Abenden dem Publikum zur Belehrung und Anregung zu dienen hatte."2

Am 19.1.1863 erfolgte die Gründung der "Mittwochs-Gesellschaft" ; Wilhelm Foerster (1832-1921), seit 1865 Direktor der Berliner Königlichen Sternwarte, gehörte der Gesellschaft von 1864 bis 1920 an. Ausgehend von seinem Engagement bei der Gründung der Astronomischen Gesellschaft schlug Eduard Schönfeld (1828-1891), Wilhelm Foersters langjähriger Freund aus Studententagen, in einem seiner Briefe an ihn im Zusammenhang mit der Benennung dieser Gesellschaft folgendes vor (3.8.1863):

"Name und Zweck: URANIA, Verein zur Förderung des Fortschritts der Astronomie im weitesten Sinne, speziell aber zur Unterstützung solcher Arbeiten, die geeignet sind, die Spezialuntersuchungen Einzelner zu erleichtern und zu fördern, und solcher, die das Material für die Untersuchungen der Zukunft liefern."

Von diesen Aktivitäten inspiriert rief Wilhelm Foerster 1887 zur Gründung der Urania-Gesellschaft auf, auch, weil der Besucheransturm die wissenschaftlichen Arbeiten der Sternwarte in den 1870er und 80er Jahren immer mehr beeinträchtigte:

"Vorschläge betreffend die Begründung einer öffentlichen teleskopischen, spektroskopischen und mikroskopischen Schaustätte zugleich zur Vorführung optischer und elektrischer Experimente sowie zu mannigfachen naturwissenschaftlichen Erläuterungen durch Wort und Bild, endlich als Ausstellungs-Ort für einschlägige Instrumente und Apparate dienend."3

So erfolgte am 3. März 1888 die Gründung der Urania-Aktiengesellschaft ${ }^{4}$ in Berlin durch Wilhelm Foerster mit finanzieller Unterstützung von Werner von Siemens (1816-1892) und Förderung durch weitere Industrielle. ${ }^{5}$ Auch

\footnotetext{
${ }^{2}$ Foerster 1913, 386. Denkschrift anläßlich des 25jährigen Bestehens der Gesellschaft "URANIA" zu Berlin, Leipzig 1913, S. 37

${ }^{3}$ Foerster 1887. Die URANIA wird in das Handelsregister des Königlichen Amtsgerichts I zu Berlin zufolge Verfügung vom 12. Mai 1888 unter Nr. 10972 eingetragen.

${ }^{4}$ Hess 1979.

${ }^{5}$ Werner von Siemens erwarb an der als Aktiengesellschaft gegründeten URANIA Anteile für 10.000 Reichsmark. Insgesamt stand der Gesellschaft URANIA ein für damalige
} 

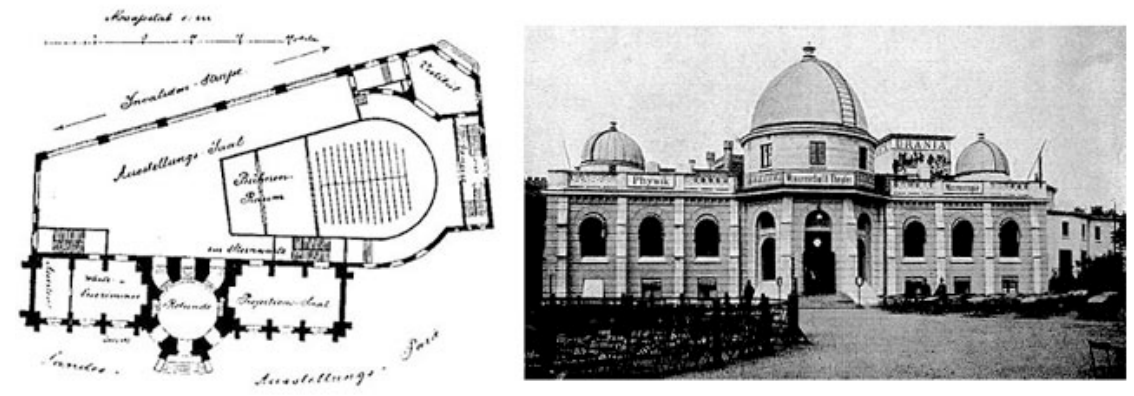

Abb. 2: Links: Grundriß Tiemann (1988), Heft 2, S. 62. Hess 1979. - Rechts: Urania, Invalidenstraße (beim Lehrter Bahnhof), Berlin, eröffnet am 1. Juli 1889

der preußische Unterrichtsminister Gustav Konrad Heinrich von Gossler (18381902) fühlte sich der "Verbreitung der Freude an der Naturerkenntnis" verpflichtet. ${ }^{6}$ Aus den Vorstellungen Foersters und Meyers entstand die Konzeption für ein Haus der Volksbildung in Berlin, bestehend aus einer Sternwarte, Experimentier-und Ausstellungssälen, dem Wissenschaftlichen Theater.

Mit Max Wilhelm Meyer ${ }^{7}$ (1853-1910) war der geeignete Mann als Leiter gefunden, der Erfahrung mit öffentlichen Vorträgen, Zeitungsartikeln und besonders in der Präsentation wissenschaftlicher Zusammenhänge auf der Bühne aus seiner Wiener Zeit aufweisen konnte.

Das Gebäude in der Invalidenstraße (beim Lehrter Bahnhof, vorher Landesausstellungspark) wurde im Juli 1888 unter Leitung von Oberregierungsrat Paul Spieker (1826- 1896) begonnen. ${ }^{8}$ Nach nur einem Jahr wurde der beeindruckende Neubau am 1. Juli 1889 feierlich eingeweiht. Das Gebäude der Urania in der Invalidenstraße gliederte sich architektonisch klar in folgende drei Teile: Den Hauptteil in Massivbauweise bildete die Sternwarte mit dem "Großen Refraktor" (12") von Carl Bamberg (1847-1892), Berlin-Friedenau, in der Hauptkuppel - ein Instrument, das damals im Deutschen Reich nur vom Straßburger Refraktor übertroffen wurde.

Verhältnisse hohes Startkapital von etwa 205.000 Reichsmark zur Verfügung.

${ }^{6}$ Denkschrift anläßlich des 25jährigen Bestehens der Gesellschaft "URANIA" zu Berlin, Leipzig 1913, S. 3.

${ }^{7}$ Meyer 1908.

${ }^{8}$ Die reinen Baukosten für das Gebäude im Landesausstellungspark beliefen sich nach Angaben von Spieker, der den Bau projektiert und dessen Ausführung in seine Regie genommen hatte, auf 180.000 Reichsmark. Allein für die Dreh-kuppel mußten 12.000 Reichsmark aufgewendet werden. Das Baugelände war der URANIA kostenlos von der preußischen Staatsregierung zur Verfügung gestellt worden. 
Daran schloß sich als zweiter Teil in Leichtbauweise eine Eisenkonstruktion für Ausstellungs-und Experimentierzwecke. Den wichtigsten Teil bildete das wissenschaftliche Theater - ebenfalls in Eisenkonstruktion. Bei der Eröffnung im Juli 1889 war die Urania in fünf Abteilungen gegliedert:

- Astronomische Abteilung (öffentliche Sternwarte, Leitung: F. Körber

- Physikalisches Kabinett, Leitung (bis 1892): Prof. E. Goldstein (18501930)

- Mikroskopische Abteilung, Leitung: William Thierry Preyer (1841-1897)

- Abteilung für Präzisionsmechanik

- Wissenschaftliches Theater $^{9}$ Leitung: Bühnenbildner und Theatermaler W. Kranz.

Zentrale Bedeutung hatte das populärwissenschaftliche Vortragswesens, dazu kamen anschauliche und experimentelle Vorführungen; es handelte sich also um eine Verschmelzung der bisherigen Betätigungen der Sternwarte und des "Wissenschaftlichen Vereins unter Hinzufügung physikalischer Veranstaltungen".

Das 19. Jahrhundert hatte, zum Beispiel mit der Anwendung von Elektrizität, zahlreiche praktisch nutzbare Wunder beschert, deren naturwissenschaftliche Voraussetzungen immer größeres Interesse erregten. Vor allem wollten die Besucher selbst, wenigstens am Modell der Maschinen, experimentieren. So wurde der Experimentiersaal schnell zum Besuchermagnet. Dieser Saal war auf Initiative des Physikers Eugen Goldstein (1850-1930) eingerichtet worden. In der URANIA gab es Wissen aus erster Hand, bedeutende Forscher und Gelehrte traten auf, oft wurde hier eine technische Neuheit der Öffentlichkeit zum erstenmal vorgestellt. Als Anfang 1890 der amerikanische Erfinder Thomas Alva Edison (1847-1931) die URANIA besuchte, schenkte er ihr zwei seiner ersten Phonographen, die lange Zeit, bis die Wiedergabe der menschlichen Stimme durch das Grammophon Allgemeingut wurde, ein besonderer Anziehungspunkt im akustischen Kabinett waren.

Meyer wiederum beabsichtigte, Bildung durch ein Wissenschaftliches Theater zu vermitteln, wie er das schon 1884 in Wien praktiziert hatte. Das wissenschaftliche Theater war dazu bestimmt,

"... ein verkleinertes Abbild der Natur, nur in ersten, allgemeinsten Umrissen, aber in möglichst wirkungsvoller Form dem unmittelbaren Verständnis einer großen Menge angepaßt und nur dem Zweck der allerersten Anregung dienend, zu entwickeln. "10

\footnotetext{
${ }^{9}$ Meyer 1892, 153. Zum Thema Naturwissenschaften im Theater gibt es aktuelle Versuche, vgl. Luehrs 2001.

${ }^{10}$ Meyer 1890a, 226 und 269.
} 


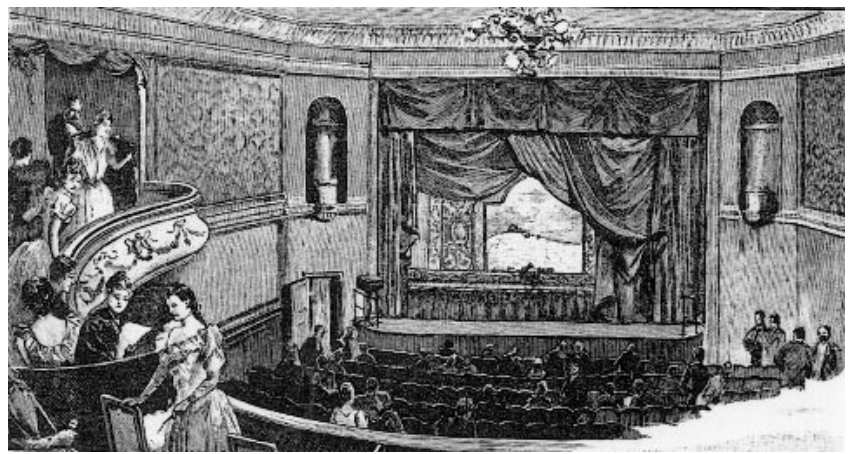

Abb. 3: Das wissenschaftliche Theater in der Urania in Berlin Meyer 1908, S. 82.

Die populären Vortragsthemen waren: "Von der Erde bis zum Mond" und "Die Geschichte der Urwelt", die auch im Ausland als Gastspiele gezeigt wurden. Vielfältige Veranstaltungenfanden inder Urania statt; ${ }^{11}$ ein Archiv von etwa 700 Dias wurde für Vortragszwecke zusammengetragen. ${ }^{12}$
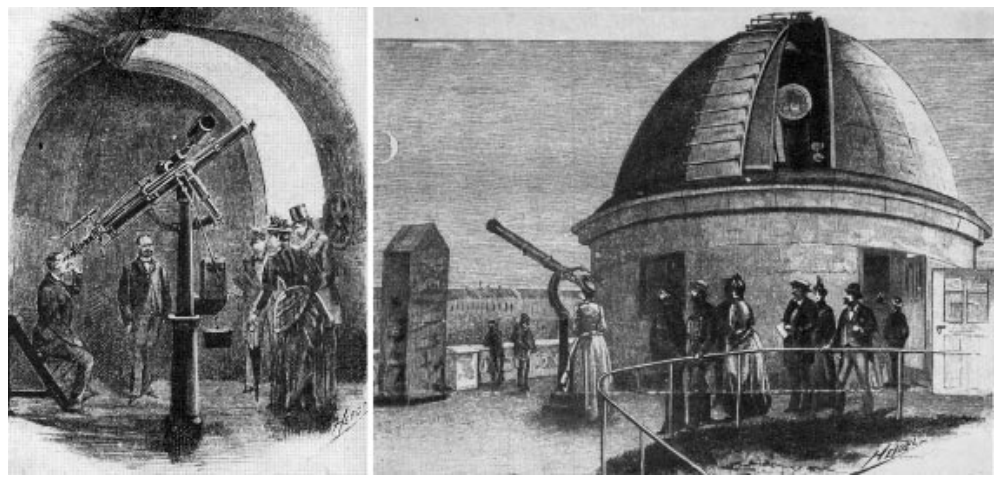

Abb. 4: Beobachtungsabend im Observatorium d. Berliner Urania 1890er Jahre Hess 1979.

Man reagierte besonders auf aktuelle Ereignisse wie Kometenerscheinungen, aber auch auf wissenschaftliche Ergebnisse; beispielsweise bewegte die Öffentlichkeit die Frage der Bewohnbarkeit des Planeten Mars; sogleich stellte Meyer in einer populären Publikation den augenblicklichen Kenntnis-

\footnotetext{
${ }^{11}$ Meyer1889.

${ }^{12}$ Meyer 1890a, 226 und 269.
} 


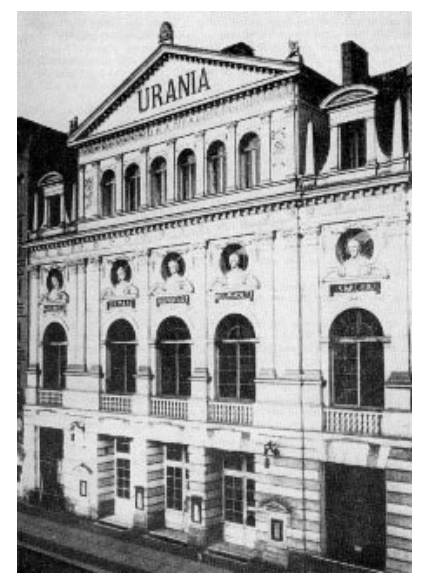

Abb. 5: Die neue Urania in der Taubenstraße 48/49 in Berlin Meyer 1908, S. 82.

stand zusammen. ${ }^{13}$ Ein Besucher beschrieb den Eindruck, den ein Abend in der Urania-Sternwarte auf ihn machte:

"Unter mir die Millionenstadt Berlin im Werktagsdunste, aus dem unzählige trübe Lichtlein emporblickten, und über mir das weite, dunkle, alles überragende und überspannende Himmelsgewölbe mit seinen feierlich funkelnden Sternen. Auf einer eisernen Wendeltreppe stieg ich von der Plattform, auf der uns kleinere, aber auch schon recht gute Fernrohre zur Verfügung standen, empor zu dem Riesenfernrohr, das durch den Schlitz einer drehbaren Kuppel wie eine mächtige Kanone in das Weltall hinausragte. Da offenbarten sich mir die Himmelswunder in ihrer ganzen Pracht und Herrlichkeit. Bald war's der Mond mit seinen Kratern und Gebirgen, bald der Jupiter mit seinen Monden oder der Saturn mit seinen Ringen, bald ein Sternhaufen mit seinem Gewimmel strahlender Sonnen, bald ein Sternennebel mit seinen chaotischen Wirbeln und Windungen!"14

Im ersten Jahr war das URANIA-Haus an 268 Tagen geöffnet, haben 98.279 Besucher 85 wissenschaftliche Vorträge und 228 Veranstaltungen im Wissenschaftlichen Theater besucht. Direktor Meyer war inzwischen als URANIAMeyer bekannt. Das Gebäude wurde diesem Besucherandrang nicht mehr gerecht. Zudem stellte sich mehr und mehr als nachteilig heraus, daß der Weg nach Moabit bei den damaligen Verkehrsverhältnissen vor allem im Winter doch recht beschwerlich war. Ein größeres und zentraler gelegenes Gebäude wurde benötigt. Am 24. April 1896 wurde in Berlin in der Taubenstraße 48/49 die neue Urania eröffnet; das alte Gebäude war zu abgelegen. Im Theater

\footnotetext{
${ }^{13}$ Meyer 1894.

${ }^{14}$ Onnasch, Karl: Wanderungen durch die Sternenwelt. Görlitz: Hoffmann \& Reiber. Zitiert nach: Friedrich 1925, S. 19.
} 
standen nun 760 Plätze statt 500 zur Verfügung. Die Fassade des imposanten Hauses war mit den Porträts von Kopernikus, Siemens, Humboldt, Helmholtz und Kepler geschmückt.

"Was einst Jules Verne [(1828-1905)] in Form des Romans gab, giebt die "Urania" in dramatischer Form": Die Bühne war ausgestattet mit modernster Bühnentechnik. "Die Phantasie wird auf das lebhafteste durch die Dekoration, durch Wandbilder und durch Beleuchtungseffekte unterstützt." Es gab im Programm: Reisen durch den Sternenraum, eine Sonnenfinsternis, Ansichten, durch welche die St. Gotthardbahn führte. Zu den Experimentalvorträgen, z.B. zu den Röntgenstrahlen oder den Hertzschen Versuchen heißt es, sie hätten auf das Publikum "wie die Premiere eines berühmten Dramatikers oder das erste Auftreten einer Diva gewirkt."15

Die Preise bewegten sich zwischen 1 Mark im zweiten Rang und 3 Mark für den Logensitz; der Eintritt in die Sammlungen kostete 50 Pfennig, ebenso die Oper (bei Benutzung der Draht-Übertragung oben im Gebäude) ${ }^{16}$ :

"Auch eine Telephonanlage befindet sich in diesem Saale, durch welche man den musikalischen Aufführungen zu folgen vermag." 17

Das Programm Meyers war hochgesteckt; er formulierte seine Ziele folgendermaßen, nämlich daß es

"...neben der vielseitigen Freude an der Naturbeobachtung und der hohen Erquickung, welche die Befriedigung der Wißbegierde auch in den einfachsten Gemüthern erweckt, den Institutionen der Urania gelingen wird, im Laufe der kommenden Jahrzehnte Viele zu erwärmen und zu erfreuen und auch einige Samenkörner auf fruchtbaren Boden auszustreuen, daß sie in selbständiger Schaffenskraft segnbringend aufkeimen ..." "...indirekt wird aber der Nutzen unserer hier flüchtig skizzierten Institutionen für die Wissenschaft unstreitig ein ganz wesentlicher werden müssen durch die lebendige, befruchtende Anregung, welche sie in jene weiten Schichten eines großen Laienpublikums streuen werden ..."19.

Die von Meyer gegründete Zeitschrift "Himmel und Erde - Illustrierte naturwissenschaftliche Monatsschrift" erschien im französischen Sprachbereich als "Ciel et Terre".

Auch in der Taubenstraße kamen bedeutende Gelehrte und Erfinder zu Wort, Namen wie Heinrich Hertz (1857-1894), Henri Poincaré (1854-1912) und Albert Einstein (1879- 1955) waren auf den Vortragslisten zu finden, auch die Polarforscher Roald Amundsen (1872-1928) und Fridtjof Nansen (1861-1930) berichteten über ihre Reisen. Der spätere Nobelpreisträger Manfred von Ar-

\footnotetext{
${ }^{15}$ Gartenlaube (1896), S. 633-637.

${ }^{16}$ Kleines Berliner Adreßbuch (1902/03).

${ }^{17}$ Gartenlaube (1896), S. 637.

${ }^{18}$ Meyer 1889, S. 31-39.

${ }^{19}$ Meyer $1890 b, 560$.
} 
denne (1907-1996) erinnerte sich viele Jahre später an die interessanten Veranstaltungen im Haus in der Taubenstraße.

Der neuen Urania war nur eine kurze Wirkungszeit unter dem schon vor dem Krieg angestrebten gemeinnützigen URANIA-Verein beschieden, der als eine Vereinigung von Freunden der Naturwissenschaft galt. Sein Anliegen war die Verbreitung und Vertiefung volkstümlicher Bildung auf naturwissenschaftlichem und technischem Gebiet. Vorsitzender wurde Franz Goerke, der von 1898 bis 1930 auch als Direktor der Gesellschaft URANIA vorstand. ${ }^{20}$ Eine bedeutende Wirkung hatte die URANIA auch auf dem Feld der Fotografie und später des Dokumentarfilms. Viele Jahre war das Haus Premiere-Filmtheater für Ufa-Dokumentarfilme. Einen Höhepunkt erlebte die Urania noch bei ihrem 25jährigen Jubiläum 1913.

Vor allem der physikalische Experimentiersaal übte erneut Faszination aus. In der Urania, im physikalischen Kabinett, eröffnete sich neben einer Ausstellung modernster Instrumente insbesondere eine Experimentiermöglichkeit, beispielsweise auf dem Gebiet der Optik und Elektrizität:

"Hier sollen Instrumente, Apparate, Einrichtungen verschiedenster Art aufgestellt werden, welche die physikalischen Erscheinungen möglichst unmittelbar verständlich darlegen. Ganz besonders soll hier Rücksicht genommen werden auf diejenigen Vorgänge, durch welche die Naturkräfte dem Getriebe des täglichen Lebens dienen [...]"21

Bezüglich der Wirkung der Urania erinnert sich Max von Laue (1879-1960) - und dies läßt sich entsprechend auf spätere naturwissenschaftlich-technische Museen - wie das Deutsche Museum - übertragen:

"Als Tertianer des Wilhelm-Gymnasiums in Berlin ... hörte ich...in der Schule ... von der Abscheidung des Kupfers aus Kupfersulfatlösungen durch den elektrischen Strom ...[Meine Mutter]... sorgte dann dafür, daß ich des öfteren in die "Urania" kam, eine populärwissenschaftliche Gesellschaft, in deren Räumen in der Taubenstraße physikalische Apparate in großer Zahl, fertig für einfache Versuche, standen; man brauchte nur nach der beigegebenen Erläuterung auf einen Knopf zu drücken und beobachtete dann einen lehrreichen Vorgang. "22

Schließlich mußte die Urania 1928 aus Finanzgründen in der Zeit der Weltwirtschaftskrise geschlossen werden. Das Haus in der Taubenstraße wurde im Zweiten Weltkrieg völlig zerstört. Auch das Gebäude mit der Sternwarte in der Invalidenstraße hatte durch Kriegseinwirkungen stark gelitten. Viele der noch intakten Instrumente waren gestohlen, einige in die Treptower Sternwarte gebracht worden. ${ }^{23}$

\footnotetext{
${ }^{20}$ Goerke, Franz: Die Urania, eine Volksbildungsstätte für Naturwissenschaften in Berlin. In: Schriften des Vereins für die Geschichte Berlins, Heft 50 (1917).

${ }^{21}$ Meyer 1889, S. 31-39.

${ }^{22}$ Laue 1961, vgl. auch Luehrs 1992.

${ }^{23}$ Entwicklung der Berliner Urania nach dem Zweiten Weltkrieg:
} 


\section{Die Wirkung der Urania}

Das Volksbildungsinstitut URANIA diente als Vorbild für ähnliche Einrichtungen in Deutschland und sogar in Europa. Speziell Wien besaß in der Volksbildung schon eine lange Tradition: Schon 1867 wurde in Wien ein Arbeiterbildungsverein gegründet.

Aus Aktivitäten in den 1880er und 1890er Jahre entstand der Volksbildungsverein als "Vorläufer und Bahnbrecher volkstümlicher Universitätskurse, jener für ganz Deutschland beispielgebenden Schöpfung Ludo Hartmanns" (18651924) [ab 1895 staatlich finanziert]. ${ }^{24}$

"Wissen ist Macht, Bildung macht frei."

Die historischen Wurzeln der Volksbildung in Österreich reichen weit in das 19. Jahrhundert zurück. Mit den erreichten Leistungen im Bereich der Demokratisierung des Zugangs zu Bildung und Wissen repräsentiert die "neutrale" Volksbildungsbewegung in exemplarischer Weise die Bemühungen um einen selbstorganisierten Abbau hierarchisch-elitärer Bildungsschranken, wie sie mit der Aufklärungstradition des reformorientierten großstädtisch-liberalen Bürgertums sowie der aufkommenden Arbeiterbewegung im 19./20. Jahrhundert gegeben sind. Im Kampf gegen obrigkeitlich-klerikale Bevormundung und im konsequenten Eintreten für Selbstbestimmung und Autonomie ("Knowledge is power", Francis Bacon (1561-1626)) stellt die Entstehungsgeschichte der politisch "ungebundenen" Volksbildung in Österreich hinsichtlich der Ideen, Akteure, Methoden und Institutionen ein integrales Element des gesellschaftlichen Modernisierungsprozesses dar. Neben der Arbeiter(kultur)bewegung, dem Austro-Marxismus, dem Wiener Kreis, der Psychoanalyse, Jugendstil und Expressionismus, zählt die Volkshochschulbewegung - um 1905 entstand in Wien das erste Abendvolkshochschulgebäude Europas - zum kulturellen Inventar der "Wiener Moderne".25

*)19.11.1953 Gründung der "Deutschen Kultur-Gemeinschaft URANIA" in Berlin (West): Der Bamberg-Refraktor, mit dem 1898 Witt den Planetoiden Eros entdeckt hatte, wurde 1948 von Amateurastronomen unter Lebensgefahr aus der zerstörten Kuppel des ersten URANIA-Hauses abgebaut und in einem zunächst provisorisch eingerichteten Observatorium aufgestellt. Heute steht das gerade erst rekonstruierte Fernrohr in der nach Wilhelm Foerster benannten Sternwarte auf dem Insulaner im Bezirk Schöneberg. Vgl. Aschenbrenner, Jutta: Bildung und die Muse der Sternenkunde. Edition Luisenstadt, Berlinische Monatsschrift Heft 7 (1998), S. 3-44, vgl. http://www.luiseberlin.de/Bms/bmstext/9807prof.htm.

*)17.6.1954 Gründungskongress der Gesellschaft zur Verbreitung wissenschaftlicher Kenntnisse im Kulturhaus "Erich Weinert" des VEB Kabelwerk Oberspree, Berlin (Ost) - die Gesellschaft geht aus dem "Kulturbund zur demokratischen Erneuerung Deutschlands" hervor.

${ }^{24}$ Reich, Emil: 25 Jahre Volksheim. Eine Wiener Volkshochschulchronik. Wien 1926. http://www.aeiou.at/aeiou.encyclop.h/h218556.htm (Dez. 2006)

${ }^{25}$ Zitiert nach: Österreichisches Volkshochschul-Archiv: http://www.vhs.or.at/archiv Mitteilung von Christian Stifter vom 18. Okt. 1999. 


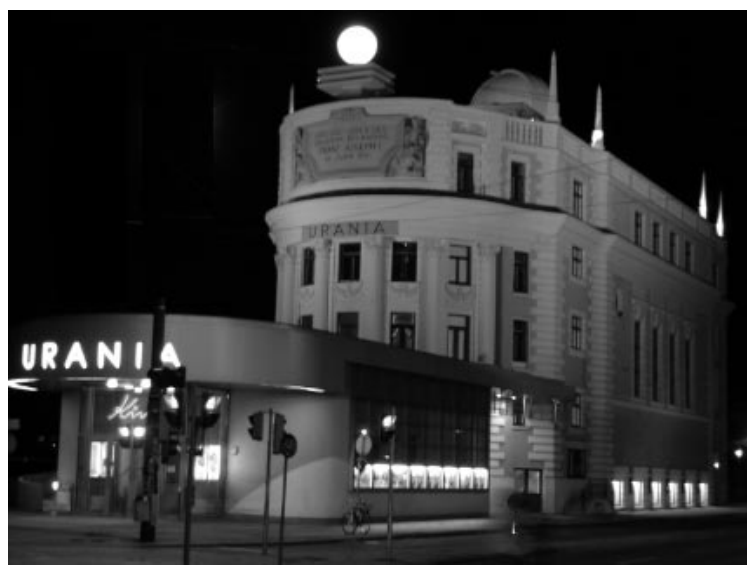

Abb. 6: Urania Wien, erbaut 1909/10 Foto: Gudrun Wolfschmidt

1897 gründete der Niederösterreichischen Gewerbeverein die Urania in Wien nach dem Berliner Vorbild als populärwissenschaftliches Institut. Bald entstand ein gemeinnütziger Verein.

1909 wurde der slowenische Architekt Max Fabiani (1865-1962), ein Schüler von Otto Koloman Wagner (1841-1918), beauftragt, für die Wiener Volkshochschule ein eigenes Bildungshaus am Donaukanal zu errichten; wichtiges astronomisches Element war die Sternwarte auf dem Dach (mit einem ZeissRefraktor) sowie die zentrale elektrische Uhrenanlage, deren Zeitzeichen auch telefonisch abgerufen werden konnten. Diese Urania setzte früh die neuesten technischen Hilfsmittel bei ihren Bildungsprogrammen ein (Lichtbild, Film). Die Einweihung der Wiener Urania in Anwesenheit von Kaiser Franz Joseph erfolgte am 20. Mai 1910. ${ }^{26}$ Folgende Neugründungen seien genannt: Magdeburg $(1894 / 1913)^{27}$, Kopenhagen (1897), Wien (1897), Budapest (1898), Zürich $(1907)^{28}$, Jena (1909), Breslau (1913), Stettin (1914), Prag (1917), Graz (1919) und in den zwanziger Jahren in Meran, Chemnitz, Moskau und Petersburg. So verbreitete sich der Urania-Gedanke.

\footnotetext{
${ }^{26}$ Der niedrige Vorbau mit der Kassenhalle wurde 1935 ergänzt. Nach Zerstörung im Zweiten Weltkrieg (1944) wurde sie 1957 wieder eröffnet und 2000/03 saniert und technisch modernisiert.

${ }^{27}$ Web-Adresse: http://www.urania-md.de/historie.html.

${ }^{28} \mathrm{http}$ // urania.astronomie.ch/, Urania-Sternwarte mit 30cm-Zeiss-Refraktor, Volkshochschule des Kantons Zürich.
} 


\section{Der Uraniagedanke}

So hat die Urania in Berlin nicht nur zu vielen weiteren Uraniagründungen - wie hier in Wien bereits 1897 - angeregt, sondern bewirkte auch die Errichtung von Volkshochschulen oder Volkssternwarten, sowie die Gründung von technischen Museen - wie des Deutschen Museums in München - mit interaktiven Experimenten. ${ }^{29}$ So trug die Urania entscheidend zur Popularisierung von Naturwissenschaft und Technik bei. Die Astronomie hatte immer eine Vorreiterrolle bei der Popularisierung.

\section{Literatur}

Foerster, Wilhelm: Vorschläge betreffend die Begründung einer öffentlichen teleskopischen, spektroskopischen und mikroskopischen Schaustätte zugleich zur Vorführung optischer und elektrischer Experimente sowie zu mannigfachen naturwissenschaftlichen Erläuterungen durch Wort und Bild, endlich als Ausstellungs-Ort für einschlägige Instrumente und Apparate dienend. Berlin: H.S. Hermann 1887.

Friedrich, Karl Josef: Deutsche Sternfreunde. Eine Blütenlese Sternenlust und Sternenweisheit. Chemnitz/Leipzig: Max Müller 1925.

Hess, Harro: Aus der Geschichte der Berliner Gesellschaft Urania (1888-1927). Vorträge und Schriften Nr. 58. Berlin-Treptow: Archenhold-Sternwarte Berlin-Terptow 1979.

Laue, Max von: Gesammelte Schriften und Vorträge. Band II. Braunschweig 1961.

Lührs, Otto: Volksbildungsinstitut Urania. In: Moebius, Hanno et al.: Vierhundert Jahre Technische Sammlungen in Berlin. Von der Raritätenkammer der Kurfürsten zum Museum für Verkehr und Technik. Berlin (Berliner Beiträge zur Technikgeschichte und Industriekultur Bd. 2) 1983.

Lührs, Otto: Vom Anschauen und Anfassen. Der Besucher als Mitspieler - Technische Mussen und ihr Wandel. In: Kultur \& Technik 3 (1992).

Meyer, Max Wilhelm: Die Veranstaltungen in der Urania. In: Himmel u. Erde 1 (1889), p. 31-39.

Meyer, Max W.: Die Urania nach ihrer Fertigstellung. In: Himmel u. Erde 2 (1890a), p. 226-269.

Meyer, Max Wilhelm: Ueber populäre Wissenschaft und Halbbildung im besonderen in bezug auf die Bestrebungen der Urania. In: Himmel und Erde 2 (1890b), p. 560.

Meyer, Max Wilhelm (Hrsg.): Illustrierter Leitfaden der Astronomie, Physik und Mikroskopie in Form eines Führers durch die Urania zu Berlin. Unter Mitwirkung von Dr. F. Körber, Prof. Dr. W. Preyer, Dr. P. Schwahn und P. Spies. Sammlung populärer Schriften herausgegeben von der Gesellschaft Urania zu Berlin, No. 12. Berlin: Hermann Paetel 1892.

Meyer, Max Wilhelm: Die physische Beschaffenheit des Planeten Mars und die Frage seiner Bewohnbarkeit nach dem Zeugniß seiner hervorragendsten Beobachter. Urania zu Berlin, No. 23. Berlin: Hermann Paetel 1894.

Meyer, Max Wilhelm: Wie ich der Urania-Meyer wurde. Eine lehrreiche Geschichte für alle, die etwas werden wollen. Hamburg: Henri Grand 1908.

Tiemann, Klaus-Harro: Die alte Berliner Urania (1888-1945). In: Urania 2 (1988), p. 62-67.

Wolfschmidt, Gudrun (Hrsg.): Popularisierung der Naturwissenschaften. Berlin, Diepholz: GNT-Verlag 2001

\footnotetext{
${ }^{29}$ Wolfschmidt 2001.
} 